\title{
WHAT TYPES OF CARS WILL WE BE DRIVING? METHODS OF FORECASTING CAR TRAVEL DEMAND BY VEHICLE TYPE
}

\author{
Tudor Mocanu \\ Deutsches Zentrum für Luft- und Raumfahrt e.V. (DLR) - German Aerospace Center \\ Institute of Transport Research \\ Rutherfordstr. 2, 12489 Berlin, Germany \\ Tel: +49 30 67055-588; Email: tudor.mocanu@dlr.de
}

Paper presented at the $97^{\text {th }}$ Annual Meeting of the Transportation Research Board TRB Paper Number: 08-05027

Initial version submitted August 1, 2017

Revised version submitted November 15, 2017

TRR final version submitted March 7, 2018

Word count: 6,497 words text +4 tables/figures x 250 words (each) $=7,497$ words 
1 ABSTRACT

2 New technologies are emerging on the private vehicle market. Conventional propulsion systems

3 are set to be replaced by alternative, more environment-friendly ones (e.g. electric vehicles), while

4 certain new features, like autonomous driving, will possibly change the way private cars are

5 employed. In order to assess the impact of such technologies, one must estimate how often and for

6 which trips these vehicle types will be used. Another issue is the exact localization of certain

7 vehicle types on the network, in order to assess environmental effects and identify where specific

8 roadside infrastructure (e.g. charging stations) will be required.

9 This paper presents four approaches on how to forecast car usage by vehicle type using a

10 macroscopic travel demand model in combination with a vehicle fleet or technology diffusion

11 model. Integrating the two types of models requires tools ranging from assumptions and

12 extrapolation of empirical data to synthetic or incremental discrete choice models. The approaches

13 are employed in a case study forecasting travel demand using privately owned autonomous

14 vehicles (AVs) in Germany in 2030. Despite identical input data, the estimated proportion of

15 vehicle miles travelled (VMT) using AVs varies between 11 and 23 percent of overall car VMT,

16 depending on the approach chosen. The reasons for this variation in results are investigated and

17 some recommendations are given. To avoid the difficulties of fitting a synthetic model to observed

18 data and increase the accuracy of the results, we recommend formulating the vehicle type choice as

19 an incremental model added to the travel demand model.

Keywords: Travel Demand Modeling, Vehicle Use, Fleet Modeling, Vehicle Technology

Diffusion, Autonomous Vehicles 
INTRODUCTION

Motorized individual transport is currently the dominant form of transportation in many Western countries and is forecasted to remain at comparably high levels in the coming decades. The expected continuity in car travel demand seems to indicate a status quo, which is far from being true. Several developments with wide-ranging implications can already be observed. The increasing focus on environmental issues spurs on the development of cleaner vehicles (e.g. electric or hybrid cars). Pollution in urban areas due to traffic-related emissions is also a growing concern and authorities are in search of policy measures to tackle this issue. Finally, a technology-driven evolution will bring new functionalities to the vehicles, the most discussed of these being automation. The emergence of autonomous vehicles (AVs) has the potential to severely influence the way private cars are used.

Understanding the relationship between the types of vehicles employed and their usage patterns is necessary to enhance forecasting in some situations where the differences between vehicles are relevant. For instance, in order to accurately model local traffic-related emissions, the traffic flows assigned to the network model should be split up according to vehicle emission profiles. Separate traffic flows for each vehicle propulsion system (e.g. petrol, diesel, electric), size, vintage etc. should be calculated. As will be shown in this paper, this modeling step is not trivial. Conversely, the emergence of new vehicle types and technologies might also have an impact on overall car travel demand. In the case of AVs, a decrease in travelers' valuation of travel time might lead to an overall increase in car travel (2). Furthermore, governments and local agencies might devise policy measures to encourage (or discourage) the use of specific vehicle types, a pertinent example being the current debate on the negative environmental effects of diesel cars in German city centers (3). Restricting diesel vehicle access in city centers would certainly have an impact on (diesel) car traffic in these areas, but it might also affect overall mode choice, destination choice and trip generation. The complex relationship between the composition of the vehicle fleet (shares of different vehicle types) and car travel demand (by vehicle type) should ideally be addressed in travel demand models (TDMs).

This paper investigates the possibility of embedding a differentiation by vehicle type into travel demand models. The objective of this paper is not to forecast the adoption of different technologies in the vehicle fleet, but rather to examine the interaction between a forecasted vehicle stock and the travel demand associated with it.

First, we present an overview of past research related to this subject. Then, the overall model framework is described and the target variables are defined. We propose four different approaches to integrating vehicle type choice into TDMs, and discuss their underlying assumptions, advantages and disadvantages. A case study, forecasting the demand for trips using private autonomous vehicles in Germany in the year 2030, is used to illustrate the different approaches. Finally, some conclusions and recommendations for further research are offered.

\section{LITERATURE REVIEW}

Research on vehicle type differentiation has been conducted since the 1970s, when the first vehicle fleet models (VFMs) were estimated (4). Such models predict long term choices made by

41 individuals or households deciding on the number and type of vehicles they own or purchase.

42 VFMs, also known as vehicle stock, holdings or car ownership models, can vary significantly in

43 terms of their complexity, ranging from simple time series to multivariate discrete choice models

44 (5). For an excellent review of state-of-the-art VFMs pertaining to the adoption of electric and

45 hybrid vehicles, see the work of Al-Alawi and Bradley ( 6 ). Other recent studies by Litman (7) and

46 Bansal and Kockelman (8) have forecasted the long-term adoption levels of AVs in the United 
States, while Kröger et al. have estimated AV adoption rates in the German car fleet using an s-shaped market-take-up vehicle technology diffusion model (9).

VFMs forecast the number and type of vehicles owned, but only rarely address vehicle usage metrics (e.g. the average number of trips per day and car, average mileage per year and car etc.). An example of a VFM also considering vehicle use is Bhat and Sen's multiple discrete-continuous extreme value (MCDEV) model (10), employed to predict both the vehicle holdings and the yearly vehicle miles of travel (VMT) per vehicle type. Bhat and Sen use the demographic characteristics of the household, vehicle specifications and land-use attributes as explanatory variables for both vehicle type ownership and use.

While such approaches may deliver aggregate results pertaining to vehicle type-specific use, they are limited in several ways. Firstly, as they do not operate with single trips or tours, they cannot link relevant trip or route attributes (e.g. distance, tolls required, available infrastructure etc.) to the use of vehicles. Secondly, they are responsive only to a limited range of policy measures, like changes in the vehicle registration fees or fuel taxes. The impact of highly localized policy tools, targeting only specific zones (e.g. city centers) or roads (e.g. special toll roads), cannot be assessed using such models. Finally, the total VMT estimated with such a model does not necessarily correspond to the VMT outputted by a dedicated TDM, making the interpretation of results difficult.

More recent studies have incorporated specific types of privately owned vehicles as an extra mode of transport in travel demand models. Auld et al. (11) and Childress et al. (12) both explored the impacts of autonomous vehicles on travel demand using activity-based models for the Chicago and Puget Sound regions respectively. Zhao and Kockelman (13) employed a macroscopic travel demand model to anticipate the impact of automated vehicles in Austin, Texas. However, the integration of VFM results into the TDM was not discussed in these approaches, with the AV penetration rates being scenario-based assumptions rather than model results.

To our knowledge, the integration of vehicle type differentiation into TDMs has not been thoroughly discussed before. Even in studies on the traffic-induced environmental effects, travel demand shares by vehicle type are either assumed (14) or not differentiated at all (15). The modeling framework proposed in this paper attempts to rectify these shortcomings.

MODELING FRAMEWORK

Overview

The aim of this paper is to present an approach to estimate car travel demand by vehicle type by integrating results from a vehicle fleet model (VFM) into a travel demand model (TDM). This implies splitting up the overall car travel demand for each origin-destination (OD) pair in the study area depending on attributes of the vehicles, the trip-maker and the trip itself. Using this setup, the model can assess the impact of a wide range of policy measures on both overall car travel demand and vehicle-type specific demand. Furthermore, as separate OD matrices for each vehicle type are generated and can be assigned to the network, this approach can also be used to forecast traffic counts by vehicle type on each road section, which is essential e.g. for fine-grained emission modeling.

For the purpose of this study, the structure and setup of the TDM are irrelevant. The model framework shown here is compatible with various macroscopic travel demand models, whether set up as traditional four-step, gravity, logit, hierarchical, simultaneous models etc. More differentiation in the TDM (e.g. by population groups/demographics, trip purposes, time of day etc.) will lead to more detailed and possibly more accurate results by vehicle type. It is worth 
33 with

noting that the approach shown here is only required when using a macroscopic TDM dealing with "person groups" or "representative persons" and not individuals or individual households. In microscopic simulations (or multi-agent tools), each individual is assigned a specific vehicle type and thus results by vehicle type can easily be aggregated rather than split up from overall car demand. However, as such models are still difficult to implement in certain large-scale applications (e.g. statewide or national models, long-term forecasting) due to computing limitations and data availability, the approach shown in this paper is (still) considered to be relevant. In the context of this paper, results from the TDM shall be noted them as $T_{i j}$, indicating a matrix with the number of car trips from origin $i$ to destination $j$.

The methodology and functionality of the VFM will not be discussed here, only its results will be considered here. Let $C_{1}, C_{2}, \ldots, C_{n}$ be vehicle categories (types) in which cars are grouped according to their attributes. Results from the VFM can be noted as the following vector of vehicle fleet shares $\boldsymbol{v s}$

$$
\boldsymbol{v} \boldsymbol{s}=\left\{v s_{C_{1}}, v s_{C_{2}}, \ldots, v s_{C_{n}}\right\}
$$

where the sum of all vehicle fleet shares equals unity.

$$
\sum_{C} v s_{C}=1
$$

Depending on the granularity of the VFM, results may be available on an individual/household level or at any superior level of aggregation (e.g. traffic zones, counties, regions etc.). For the sake of simplicity, the indices denoting the spatial distribution of the vehicle fleet shares will be omitted in this paper.

The target variable is defined as the proportion of car trips using a vehicle from category $C$, and will be noted as the (matrix) vector

$$
\boldsymbol{u} \boldsymbol{s}_{i j}=\left\{u s_{i j, C_{1}}, u s_{i j, C_{2}}, \ldots, u s_{i j, C_{n}}\right\}
$$

where the sum of all usage shares also equals unity.

$$
\sum_{C} u s_{i j, C}=1
$$

Note that unlike the vehicle fleet shares $\boldsymbol{v s}$, which denote attributes of the population and are only influenced by the home location of the travelers, the usage shares might be different for each origin-destination (OD) pair $i j$ and should therefore be noted as a vector $\boldsymbol{u} \boldsymbol{s}_{i j}$. In other words, vehicles will not automatically be used in equal proportion for all trips.

Multiplying the usage shares of a vehicle category with the total car trip matrix $T_{i j}$ then gives the trip matrix for that vehicle category:

$$
T_{i j, C}=u s_{i j, C} T_{i j}
$$

$$
\sum_{C} T_{i j, C}=T_{i j}
$$

The key in determining car travel demand by vehicle type is forecasting the usage shares vector 
$\boldsymbol{u} \boldsymbol{s}_{i j}$, as this information is not included in either the TDM or the VFM alone. Using the vehicle fleet shares $\boldsymbol{v s}$ from a VFM as input, there are several methods of estimating $\boldsymbol{u} \boldsymbol{s}_{i j}$. Four of these, numbered A1 to A4, will be discussed in the following section. Depending on the model application and data availability, each one of them might seem attractive to use at some point. However, as will be shown in the case study, results might greatly differ, so the adequacy of the method employed must be well weighted.

\section{Approaches to Estimating Vehicle Usage Shares}

\section{A1. Trivial Approach}

A simple approach to link the results from the TDM and VFM would be to consider that the vehicle usage shares are equal to the vehicle fleet shares (obtained from the VFM) for all OD pairs:

$$
\begin{gathered}
\boldsymbol{u s _ { i j }}=\boldsymbol{v} \boldsymbol{s} \\
T_{i j, C}=u s_{i j, C} T_{i j}=v s_{C} T_{i j}
\end{gathered}
$$

A1 works under the assumption that all vehicles are used equally often, independent of any attribute of the vehicle itself (e.g. size, comfort, fuel costs etc.) or of the trip undertaken (e.g. trip purpose, distance, travel time etc.). The obvious advantage of A1 is given by its simplicity and ease of use. This approach does not require any additional data and there are no model parameters to be estimated or calibrated.

Results using A1 will be unrealistic whenever there is good reason to assume that certain car attributes are correlated to specific usage statistics. For instance, the average annual mileage of a petrol-fueled car in Germany amounts to ca. $10,000 \mathrm{~km}$, while diesel vehicles are driven on average ca. 17,000 km (own analyses on national household survey data). In this case, the assumption of equal usage would obviously lead to wrong forecast results. An argument could be made that the independence of the choice outcome with respect to vehicle and trip attributes is indeed given for a large proportion of the population - those individuals or households that only possess one vehicle and thus cannot choose between multiple vehicles for each trip. However, it is not unreasonable to assume that these decision makers will consider their predicted annual mileage when buying a car, thus correlating the available vehicles to their travel patterns. Therefore, while A1 does deliver a very rough estimation of vehicle usage shares, it is not suited for precise forecasting and should rather be used as a benchmarking tool for the more complex approaches shown in the following sections.

\section{A2. Trip Segmentation Approach}

The Trip Segmentation Approach (A2) derives the usage shares from observed data in the base year. The concept revolves around the idea that, by segmenting trips into homogenous groups, all relevant differences in the car usage patterns can be identified. Assuming that all relevant explanatory factors have been identified in the base year analysis, the empirically derived usage shares can then also be used for forecasting.

In order to provide statistically reliable results, comprehensive data on the vehicle usage in the base year is required. Ideally, a trip database should be available containing information on the trip itself, as well as on the characteristics of the vehicle used. Regional or national household surveys should provide this information for a sufficiently large number of trips. For instance, the German national household survey (I) reports on ca. 70,000 car trips and the corresponding ca. 
35,000 vehicles used on these trips.

2 TABLE 1 Observed Vehicle Usage Shares in Germany

\begin{tabular}{|c|c|c|c|c|c|c|c|c|c|c|c|c|c|c|}
\hline \multirow[b]{2}{*}{ Distance } & \multirow[b]{2}{*}{ Purpose } & \multicolumn{3}{|c|}{ Small } & \multicolumn{3}{|c|}{ Compact } & \multicolumn{3}{|c|}{ Mid-Size } & \multicolumn{3}{|c|}{ Large } & \\
\hline & & $<4 \mathrm{yrs}$ & 4-8yrs & $>8 y r s$ & $<4 y r s$ & $4-8 y r s$ & $>8 y r s$ & $<4 \mathrm{yrs}$ & $4-8 y r s$ & $>8 y r s$ & $<4 \mathrm{yrs}$ & $4-8 y r s$ & $>$ 8yrs & \\
\hline \multirow{3}{*}{$<20 \mathrm{~km}$} & Commute & 0.072 & 0.078 & 0.133 & 0.104 & 0.096 & 0.167 & 0.092 & 0.089 & 0.154 & 0.005 & 0.002 & 0.010 & 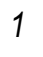 \\
\hline & Business & 0.059 & 0.051 & 0.089 & 0.112 & 0.116 & 0.159 & 0.148 & 0.117 & 0.120 & 0.006 & 0.009 & 0.015 & 1 \\
\hline & Other & 0.067 & 0.069 & 0.097 & 0.117 & 0.104 & 0.162 & 0.098 & 0.107 & 0.165 & 0.003 & 0.003 & 0.007 & 1 \\
\hline \multirow{3}{*}{$20-50 \mathrm{~km}$} & Comr & 0.085 & 0.081 & 0.104 & 0.115 & 0.118 & 0.155 & 0.097 & 0.099 & 0.131 & 0.004 & 0.004 & 0.006 & 1 \\
\hline & Busine & 0.057 & 0.075 & 0.057 & 0.159 & 0.062 & 0.115 & 0.229 & 0.097 & 0.137 & 0.009 & 0.000 & 0.004 & 1 \\
\hline & Other & 0.070 & 0.069 & 0.080 & 0.119 & 0.102 & 0.172 & 0.114 & 0.114 & 0.151 & 0.002 & 0.002 & 0.005 & 1 \\
\hline \multirow{3}{*}{$50-100 \mathrm{~km}$} & Commute & 0.062 & 0.057 & 0.084 & 0.160 & 0.135 & 0.116 & 0.114 & 0.113 & 0.138 & 0.000 & 0.003 & 0.017 & 1 \\
\hline & Business & 0.060 & 0.060 & 0.036 & 0.131 & 0.071 & 0.083 & 0.262 & 0.119 & 0.119 & 0.012 & 0.036 & 0.012 & 1 \\
\hline & Other & 0.076 & 0.069 & 0.057 & 0.116 & 0.079 & 0.169 & 0.113 & 0.143 & 0.146 & 0.004 & 0.008 & 0.019 & 1 \\
\hline \multirow{3}{*}{$100-200 \mathrm{~km}$} & Commute & 0.107 & 0.019 & 0.049 & 0.107 & 0.097 & 0.136 & 0.252 & 0.087 & 0.136 & 0.000 & 0.010 & 0.000 & 1 \\
\hline & Business & 0.050 & 0.033 & 0.017 & 0.150 & 0.083 & 0.050 & 0.283 & 0.083 & 0.183 & 0.050 & 0.017 & 0.000 & 1 \\
\hline & Other & 0.060 & 0.060 & 0.044 & 0.129 & 0.096 & 0.107 & 0.151 & 0.159 & 0.173 & 0.011 & 0.005 & 0.005 & 1 \\
\hline \multirow{3}{*}{$>200 \mathrm{~km}$} & Commute & 0.057 & 0.068 & 0.034 & 0.080 & 0.148 & 0.136 & 0.239 & 0.068 & 0.125 & 0.034 & 0.000 & 0.011 & 1 \\
\hline & Business & 0.000 & 0.000 & 0.022 & 0.200 & 0.000 & 0.044 & 0.467 & 0.089 & 0.156 & 0.000 & 0.022 & 0.000 & 1 \\
\hline & Other & 0.057 & 0.049 & 0.036 & 0.166 & 0.117 & 0.101 & 0.178 & 0.117 & 0.162 & 0.004 & 0.000 & 0.012 & 1 \\
\hline \multicolumn{2}{|c|}{ Vehicle fleet shares } & 0.067 & 0.075 & 0.121 & 0.104 & 0.097 & 0.185 & 0.083 & 0.089 & 0.160 & 0.004 & 0.003 & 0.011 & . \\
\hline
\end{tabular}

TABLE 1 exemplifies an analysis using the Trip Segmentation Approach on German data. Car trips are grouped according to four distance categories and three trip purposes, while vehicles are differentiated according to their size and age. Each row shows the vehicle usage shares $\boldsymbol{u s}_{i j}$ for that particular trip category. The vehicle fleet shares $\boldsymbol{v s}$ (proportion of vehicles in a category from the total car fleet) are also given at the bottom of the table for comparison.

The empirical data in TABLE 1 indicates that trip purpose and distance are not independent of the size and age of the vehicle employed. For short trips, the usage shares closely resemble the vehicle fleet shares, suggesting that for these trips, car attributes have little explanatory power.

11 This is exactly the assumption followed by the Trivial Approach (A1). For longer trips, however, 12 the usage shares of both larger and newer vehicles increase. This is particularly evident for business trips, where the disparity between the usage and vehicle fleet shares is the largest. As long trips have a significantly higher impact on the overall VMT, this also shows the limitations of A1.

Using the Trip Segmentation Approach for forecasting is straightforward. Each OD relation from the forecasted overall car travel matrix is split up according to the corresponding trip category usage shares $\boldsymbol{u} \boldsymbol{s}_{i j}$ from TABLE 1 . If the trip structure in the forecast is different from the base year (e.g. higher proportion of long trips, other trip purposes etc.), then different overall car usage shares will result.

The Trip Segmentation Approach is more complex than A1 and delivers more accurate th. This improvement comes at the price of increased data requirements. A comprehensive database containing observed trips and vehicles is necessary. In the absence of sufficient trip observations in the database, segmentation by too many criteria can lead to statistically unreliable 
usage shares.

A difficulty using A2 arises when forecasting the usage of vehicle types that will only become available in the future and for which there is no empirical usage data available. Another drawback of A2 is given by the fact that the future usage shares are not responsive to changes in the forecasted vehicle fleet shares (distribution of car types by age, size etc.). For instance, with this approach, an increase in the proportion of "large and new" vehicles would not lead to higher vehicle usage shares for these cars. In order to make the results responsive to multiple factors, a more complex modeling approach is required.

\section{A3. Choice Model Approach}

Mocanu and Winkler (16) present an approach to differentiate car travel demand using the discrete choice model framework. They propose splitting results from the TDM into different vehicle categories using a nested logit-structured model extension. This model setup adds car type choice at the lowest level of the TDM choice hierarchy. Thus, a two-level nested logit is created, where destination and mode choice are calculated in the upper nest and vehicle type choice is calculated in the lower nest.

The choice of vehicle type in the lower nest is formulated as a standard multinomial logit (MNL), with the usage shares vector $\boldsymbol{u} \boldsymbol{s}_{i j}$ resulting from the individual utilities $V$ of each vehicle category $C$ :

$$
u s_{i j, C}=\frac{\exp \left(\mu V_{i j, C}\right)}{\sum_{C} \exp \left(\mu V_{i j, C}\right)}
$$

where $\mu$ defines the logsum scale parameter of the (lower) vehicle type choice nest.

The utilities $V$ are given as a function of the vehicle fleet shares $\boldsymbol{v s}$ and all other relevant variables, e.g. travel time, access and egress time, cost etc.

$$
V_{i j, C}=f\left(v s_{C}, t t_{i j, C}, c_{i j, C}, \ldots\right)
$$

Using the model structure proposed by Mocanu and Winkler, it is possible to fully embed the vehicle type choice in the TDM and also to create a link back from the vehicle type choice to the TDM using the logsum (also called inclusive value or maximum expected utility) over all available alternatives. The authors show that, despite linking up the two models using the nested logit framework, the model can be implemented independently of the original TDM setup and the required modifications to the TDM are minimal.

The Choice Model Approach enables the entire TDM to be responsive to measures that only affect some vehicle types. Imagine a policy measure like a pollution tax for city centers, applicable only to conventionally-fueled vehicles (but not to electric cars). The model should not only forecast a decreasing number of car trips using conventional vehicles in city centers, but also a general reduction of car traffic (in those areas), changes in the destination choice and possibly also changing trip rates. Conversely, adding a very attractive vehicle type (e.g. AVs) will increase the car logsum and influence mode choice, destination choice and trip generation, thus creating the expected induced traffic. Using forms of composite costs over all vehicle types other than the logsum (e.g. simple or weighted averages) might lead to undesired model results, as was shown for instance by Williams as early as 1977 (17).

One issue with A3 is the fact that this choice model is only a hypothetical construct, due to 
two reasons. On the one hand, vehicle purchase and ownership are long-term decisions, while vehicle type usage for each trip is a short-term decision. On the other hand, not all individuals have the freedom of choosing between all vehicle types for each of their trips. As each household owns only a limited number of cars, the long-term ownership decision restricts the available choice set in the short-term decision. Consequently, separate models should be set up for each combination of available vehicles. Due to the large number of possible vehicle type ownership combinations and the ensuing computing time and number of parameters required, such a model setup is not feasible. Instead, restrictions in the available choice set have to be applied aggregately on only one model using the vehicle fleet shares vs, as discussed by Mocanu and Winkler (16).

This implementation of a discrete choice model has wide-ranging implications for the estimation of preferences and model parameters. Because individual choices, as modeled, cannot be observed in reality, a model estimation using standard techniques is virtually impossible. Stated preference experiments and revealed preference data cannot simulate sufficient variation in the input variable $\boldsymbol{v s}$, while at the same time keeping all other variables unchanged, in order to estimate the impact of this variable on utility. Therefore, the calibration of the vehicle type choice parameters, and also the logsum scale parameter $\mu$, must occur heuristically.

Due to the difficulties in estimating the vehicle type choice parameters, it becomes evident that the more vehicle categories and differentiation criteria are considered, the harder it is to fit such a model to observed data. Furthermore, it is not only important to replicate observed car usage patterns, but also to ensure that the choice model reacts in a predictable manner to variable input and exhibits plausible elasticities and substitution patterns. This is especially difficult when differentiating vehicles by multiple criteria (e.g. propulsion system and size), as this setup obviously violates the MNL assumption of independently distributed error terms. Under these circumstances, formulating and estimating a vehicle type choice model to fit the data in TABLE 1 is no trivial task.

\section{A4. Incremental Approach}

Both the Trip Segmentation Approach (A2) and the Choice Model Approach (A3) can deliver plausible results for the differentiation of car travel, but both also have significant drawbacks and weaknesses. A2 is based on the assumption that the observed vehicle type usage will remain unchanged for similar trips, while A3 has difficulties in reproducing the observed data if the vehicle classification is too complex.

The goal of the Incremental Approach (A4) is to enhance the Trip Segmentation Approach with the advantages offered by the Choice Model Approach. Observed vehicle type usage preferences should be reproduced in the base year, while at the same time making the choice sensitive to variable input. This is possible by setting up the vehicle type choice as an incremental (or pivot-point) choice model, as described for instance in (18). The forecasted vehicle type usage shares vector $\boldsymbol{u s} \boldsymbol{s}_{i j}^{F}$ will then be:

$$
u s_{i j, C}^{F}=\frac{u s_{i j, C}^{B Y} \exp \left(\mu\left(V_{i j, C}^{F}-V_{i j, C}^{B Y}\right)\right)}{\sum_{C} u s_{i j, C}^{B Y} \exp \left(\mu\left(V_{i j, C}^{F}-V_{i j, C}^{B Y}\right)\right)}
$$

where $u s_{i j, C}^{B Y}$ denotes the observed vehicle type usage shares from the base year, as exemplified in TABLE 1 , and $V_{i j, C}^{F}, V_{i j, C}^{B Y}$ the utilities of vehicle category $C$ in the forecast and base year

41 respectively. 
This model formulation presents several advantages compared to A3. Firstly, the observed usage shares can be replicated exactly, independent of the formulation of utility $V_{i j, C}$. Running a "forecast" under the assumption that $V_{i j, C}^{F}=V_{i j, C}^{B Y}$ is exactly what is assumed in A2 and leads to $u s_{i j, C}^{F}=u s_{i j, C}^{B Y}$. Therefore, A4 is a more general formulation of A2. Secondly, if the vehicle fleet share $v s_{C}$ is incorporated into the utility $V_{i j, C}$, the model results become responsive to changes in the vehicle fleet structure. The lack of such sensitivity was mentioned above as one of the major limitations of A2. Finally, as only differences in utility are relevant, the model can be specified with fewer variables than A3. This should simplify the process of parameter estimation and make the assessment of elasticities and overall model responsiveness easier to handle. Nevertheless, the difficulties in estimating the model parameters mentioned in the previous section also apply to A4.

\section{CASE STUDY}

\section{Scope}

13 The following case study will be used to exemplify the modeling framework proposed in this paper. The primary goal of the study is to compare the differences in the four approaches presented above. The object of the study is a forecast of travel demand by autonomous vehicles (AV) in Germany in 2030. The relevant measure of travel demand considered here is VMT per average working day.

The aim of the case study is not to comprehensively analyze all aspects related to the future development of demand for AVs in Germany. There are many factors of technical, behavioral and legal nature not considered in this case study. The focus here lies more on the methodology and less on the actual results. In order to simplify the model implementation and the interpretation of results, the following restrictions apply:

- Only commuting trips are considered.

- Only privately owned vehicles are considered (no car sharing, vehicles on demand, robotaxis, car rentals etc.).

- AV are considered to be only those vehicles with full automation (e.g. Highway Pilot technology, Level 4 and above), all other vehicles are defined as non-autonomous (non-AV).

- Empty AV trips are not considered in the VMT calculations.

- Overall mode and destination choice is not affected by the vehicle type choice; hence the overall number of car trips and car VMT is equal in all approaches.

The last bullet point on the list is necessary in order to enable a comparison between those approaches that can model the effects on mode and destination choice (A3 and A4) and those that cannot (A1 and A2). Furthermore, in order to exemplify the impact that user preferences have on the vehicle type usage, we also assume that AVs lead to a decrease in the users' valuation of travel time savings (vtts), as the in-vehicle time can also be used for other activities instead of driving. The vtts reduction assumed is 25 percent starting with the eleventh minute of travel on, following the results of an extensive literature study (9). Note that the vtts reduction only affects the choice between $\mathrm{AV}$ and non-AV and not the overall mode and destination choice, once again in order to enable the comparison between the four approaches. 


\section{TDM and VFM input}

2 Total car travel demand for Germany was leveraged from the DEMO national travel demand

3 model (19) in form of trip matrices $T_{i j}$. For the purpose of this case study, only the results pertaining to commuting trips are relevant. TABLE 2 shows the forecasted overall number of car trips and VMT.

TABLE 2 Forecasted Overall Car Travel Demand in Germany, 2030

\begin{tabular}{l|l} 
& (commuting trips only) \\
\hline Total number of car trips per average working day & $30.5 \mathrm{~m}$ trips \\
Total car VMT per average working day & $568.1 \mathrm{~m}$ vehicle $\mathrm{km}$ \\
Average trip length & $18.6 \mathrm{~km}$ per trip
\end{tabular}

A VFM was used to forecast the shares of autonomous vehicles in the German fleet. The model setup is briefly presented in (9) and uses an s-shaped market-take-up curve to model the diffusion of automation technologies. Vehicles were grouped into four size categories in order to enable different technology diffusion rates, while vehicle vintage is a model result. In this case study we assumed that AV technology will first be available for large vehicles starting with 2020 and subsequently also for the smaller car segments. TABLE 3 shows the main results from the VFM.

TABLE 3 Forecasted Vehicle Fleet Shares in Germany, 2030

\begin{tabular}{l|cc|c|r} 
& AV & non-AV & $\boldsymbol{\Sigma}$ & AV available starting \\
\hline Small & 0.000 & 0.227 & 0.227 & n.a. \\
Compact & 0.033 & 0.310 & 0.343 & 2024 \\
Mid-Size & 0.075 & 0.346 & 0.421 & 2021 \\
Large & 0.004 & 0.005 & 0.009 & 2020 \\
\hline $\boldsymbol{\Sigma}$ & 0.112 & 0.888 & 1.000 &
\end{tabular}

\section{Implementation of the Linking Approaches}

\section{$15 \quad A 1$}

16 The implementation of the Trivial Approach is straightforward. AV usage shares for all trips result from the vehicle fleet shares given in TABLE 3, meaning that irrespective of trip purpose and distance the share of AV trips from all car trips will be 11.2 percent.

A2

The Trip Segmentation Approach used the analysis shown in TABLE 1. The German car fleet was segmented into the 12 categories shown (four size $\mathrm{x}$ three age categories). The vehicle usage shares given in TABLE 1 were applied to the forecasted car travel demand matrix, thus obtaining the usage share of each of the 12 vehicle categories for every OD relation.

In order to estimate demand for autonomous vehicles, the assumption was made that for each of the 12 vehicle categories, AV and non-AV usage shares are equal to the vehicle fleet shares in that category. This implies that usage preferences for new and large AV are equal to those for new and large non-AV, and so on. Thus, differences in the utilization of AV and non-AV only result from the car size and age. 
A3

In order to illustrate the Choice Model Approach, a simple model was set up with two vehicle categories: autonomous ( $A V)$ and non-autonomous ( $n A V)$ vehicles. The utility function for these vehicle categories was adopted from the German National TDM (10). This simplified utility function takes the linear form:

$$
V_{i j}=\beta_{v s} \ln (v s)+\beta_{t t} t t_{i j}+\beta_{a e t} a e t_{i j}+\beta_{c}\left(f c_{i j}+p c_{i j}\right)
$$

where $t t_{i j}$ denotes travel time, $a e t_{i j}$ access and egress time, $f c_{i j}$ fuel costs and $p c_{i j}$ parking fees.

8 Note that car size and age do not enter the utility function. The differences in AV and non-AV

9 utility are given only by the vehicle fleet shares and the reduced valuation of travel time. As only

10 differences in utility are relevant in the logit model setup, with the logsum scale parameter $\mu=1$

11 and the vehicle fleet shares parameter $\beta_{v s}=1$ the resulting vehicle usage shares can be formulated

12 as follows:

$$
u s_{i j, A V}=\frac{v s_{A V} \exp \left(f\left(t t_{i j}\right)\right)}{v s_{A V} \exp \left(f\left(t t_{i j}\right)\right)+v s_{n A V} \exp \left(\beta_{t t} t t_{i j}\right)}
$$

$$
u s_{i j, n A V}=\frac{v s_{n A V} \exp \left(\beta_{t t} t t_{i j}\right)}{v s_{A V} \exp \left(f\left(t t_{i j}\right)\right)+v s_{n A V} \exp \left(\beta_{t t} t t_{i j}\right)}
$$

As the utility function $V$ is linear in travel times and costs, the vtts reduction by 25 percent from

$$
f\left(t t_{i j}\right)=\max \left\{\beta_{t t} t t_{i j}, \frac{\beta_{t t}}{1.25}\left(t t_{i j}[\min ]-10 \min \right)+\beta_{t t} 10 \min \right\}
$$

The travel time parameter used for this case study was $\beta_{t t}=-0.06$.

A4

The Incremental Approach employs the vehicle usage shares from TABLE 1 as base year values in an incremental (pivot-point) model. Each of the vehicle categories was split into two corresponding vehicle types, with and without autonomous technology respectively. The vehicle fleet shares $\boldsymbol{v} \boldsymbol{s}^{B Y}$ and usage shares $\boldsymbol{u} \boldsymbol{s}^{B Y}$ in the base year are considered to be 0 for $\mathrm{AV}$ and 1 for non-AV for all vehicle size and age categories, as this technology does not yet exist in the base year. For the forecast, the VFM provides the vehicle fleet shares $\boldsymbol{v} \boldsymbol{s}^{F}$, as given in TABLE 3 . The utility formulation is identical to the implementation of $\mathrm{A} 3$, including the parameters and the reduction of travel time valuation shown in eq. (10). Thus, the incremental model for each vehicle category can be formulated as:

$$
u s_{i j, A V}^{F}=\frac{v s_{A V}^{F} \exp \left(f\left(t t_{i j}\right)-\beta_{t t} t t_{i j}\right)}{v s_{A V}^{F} \exp \left(f\left(t t_{i j}\right)-\beta_{t t} t t_{i j}\right)+v s_{n A V}^{F}}
$$




$$
u s_{i j, n A V}^{F}=\frac{v s_{n A V}^{F}}{v s_{A V}^{F} \exp \left(f\left(t t_{i j}\right)-\beta_{t t} t t_{i j}\right)+v s_{n A V}^{F}}
$$

6 in eq. (11.1) and (11.2).

\section{Results and Interpretation}

and shares.

$$
\lim _{u s_{A V}^{B Y}, v s_{A V}^{B Y} \rightarrow 0} \frac{v s_{A V}^{F} u s_{A V}^{B Y}}{v s_{A V}^{B Y}}=v s_{A V}^{F}
$$

TABLE 4 gives an overview of the aggregated results obtained with the four segmentation approaches. The number of AV and non-AV trips is given, as well as overall VMT and the ensuing average trip distances. The differences in results are significant, particularly regarding the VMT

\begin{tabular}{|c|c|c|c|c|c|c|c|c|}
\hline & \multicolumn{2}{|c|}{$\begin{array}{c}\text { Total number of car } \\
\text { trips } \\
{[\mathrm{m}]}\end{array}$} & \multicolumn{2}{|c|}{$\begin{array}{l}\text { Total car VMT } \\
\text { [m veh. km] }\end{array}$} & \multicolumn{2}{|c|}{$\begin{array}{l}\text { Average car trip } \\
\text { length } \\
{[\mathrm{km}]}\end{array}$} & \multicolumn{2}{|c|}{ considers higher AV usage due to } \\
\hline & AV & non-AV & AV & non-AV & $\mathrm{AV}$ & non-AV & $A V$ size and age & vtts reduction \\
\hline A1 & 3.4 & 27.1 & 63.7 & 504.4 & 18.6 & 18.6 & not & not \\
\hline A2 & 4.8 & 25.7 & 102.1 & 466.0 & 21.1 & 18.1 & yes & not \\
\hline A3 & 3.9 & 26.7 & 84.5 & 483.6 & 21.9 & 18.1 & not & yes \\
\hline A4 & 5.5 & 25.1 & 130.5 & 437.6 & 23.9 & 17.5 & yes & yes \\
\hline
\end{tabular}

\section{TABLE 4 Overview of the Case Study Results}

13 A1 forecasts the lowest share of trips and VMT using AV. In contrast to the other approaches, with

14 A1 there is no reason why autonomous cars should be used more often than non-AVs. The vehicle 15 usage shares are equal for all trips, thus leading to an equal average trip distance for AVs and

16 non-AVs. Furthermore, the assumed reduction in the valuation of travel time cannot be considered 17 using this approach.

A2 forecasts a significantly higher share of AV trips and VMT. This result is due to VFM results showing that autonomous cars are larger and newer than the average car, and observed data shows such vehicles are being used more often and for longer trips. This also explains the longer average AV trip distances. The Trip Segmentation Approach, however, cannot consider the additional assumption of reduced vtts for AVs. If differentiated results for each vehicle category (grouped by size and age as in TABLE 1) were shown, usage shares and trip distances for autonomous and non-autonomous vehicles would be equal inside each category.

A3 also predicts more AV trips and VMT than A1. This approach considers the relative advantage of AVs due to the assumed vtts reduction, thus leading to more AV trips and longer average trip distances. However, A3 does not take into consideration that AVs are more often 
larger and newer vehicles, which have been shown to be used relatively more often, irrespective of whether they have or do not have any automation technology onboard.

Finally, A4 attempts to consider both AV comparative advantages and thus leads to the highest number of AV trips and VMT. Compared to A2, average AV trip distances increase by a further $2.8 \mathrm{~km}$ due to the vtts assumptions.

The results in TABLE 4 suggest that A1 is only suited for very rough first estimations. If the vehicle usage differs considerably between the vehicle categories, then A1 cannot deliver accurate results. A comparison between A2 and A3 is difficult, as in their implementation in the case study shown above they both focus on different usage aspects. Ideally, a discrete choice modeling approach could and should also incorporate vehicle size and age in the utility function, which would make the results easier to compare to A2. If all relevant input data was captured in such an ideal model, one would expect the results to be similar to those obtained using A4. The problem is that such a model is difficult to formulate and estimate, as discussed in the section pertaining to the Choice Model Approach. Thus, further research is required to enhance the discrete choice modeling approach.

A4 offers an attractive and apparently easy to implement option of incorporating the advantages of the other two approaches, and would seem to deliver the most plausible results in this case study. However, this hybrid empirical and choice model construct should be studied further and implemented in other case studies as well in order to better understand the overall model responsiveness and the predictability of results.

The case study shown here does not contain any spatially heterogeneous policy measures, which would not affect car travel demand uniformly over the entire OD matrix, but only influence certain OD pairs. Such measures cannot be adequately assessed using A1 or A2. A modeled approach like A3 or A4 would be necessary to assess such scenarios. Furthermore, overall mode and destination choice was kept unchanged in the four approaches even though A3 and A4 offer the possibility of also estimating induced traffic. Enabling this feature will likely further increase the AV VMT shares using A3 and A4.

\section{CONCLUSIONS AND OUTLOOK}

The work presented in this paper is driven by the need to better forecast the impact of new technologies on motorized individual transport demand. As vehicle attributes are correlated to usage patterns, it becomes necessary to differentiate car travel demand according to these attributes. This becomes even more important when assessing the environmental effects of car traffic.

The aim of this paper was to present a modeling framework for splitting up overall car travel demand originating from a macroscopic travel demand model. A necessary prerequisite for this method is the existence of an independent vehicle fleet model forecasting the composition of the car fleet. Four approaches to linking the results from these two models were discussed.

Apart from the trivial solution of splitting up demand according only to the shares of respective vehicles in the fleet, the other three approaches all have their merits, but also their shortcomings. The Trip Segmentation Approach relies on observed usage patterns and assumptions and is therefore not sensitive to modifications in the input parameters. The Choice Model Approach is versatile, but the logit model parameters leading to a good fit to observed data are difficult to estimate. Finally, the Incremental Approach attempts to combine the advantages of the other approaches, but its responsiveness to variable input is somewhat difficult to ascertain.

Results obtained using the four approaches were compared in a case study forecasting demand for travel with private autonomous vehicles in Germany in the year 2030. The results 
1 showed significant differences, particularly in the prediction of AV VMT. As expected, the 2 forecasted demand for AV travel was lowest using the Trivial Approach and highest using the 3 Incremental Approach.

4 In the case study shown, the Discrete Choice Approach was implemented using a very 5 simple model formulation. Further work is required to enhance the utility function by also 6 incorporating other vehicle attributes. It is expected that an ideally formulated and calibrated

7 Discrete Choice Approach should lead to similar results to the Incremental Approach. The 8 theoretical and practical difficulties related to the model estimation require further analyses.

9 Until a more complex Discrete Choice Approach can be implemented, the Incremental 10 Approach seems to be a better suited method of linking macroscopic travel demand and vehicle 11 fleet models. The approach should be put to use in other case studies as well, in order to better 12 understand the way it responds to different input data. 


\section{REFERENCES}

[1] infas, and DLR. Mobilität in Deutschland 2008: Ergebnisbericht. Bonn und Berlin, 2010.

[2] Fagnant, D. J., and K. Kockelman. Preparing a nation for autonomous vehicles: opportunities, barriers and policy recommendations. Transportation Research Part A: Policy and Practice, Vol. 77, No. Supplement C, 2015, pp. 167-181.

[3] Reuters. German court backs bid to ban diesel cars in Stuttgart. https://www.reuters.com/article/us-germany-emissions-idUSKBN1AD10Y. Accessed 01.08.2017.

[4] Lave, C. A., and K. Train. A disaggregate model of auto-type choice. Transportation Research Part A: General, Vol. 13, No. 1, 1979, pp. 1-9.

[5] Zhao, Y., and K. M. Kockelman. Household vehicle ownership by vehicle type: Application of a multivariate negative binomial model. Presented at Transportation Research Board 81st Annual Meeting, Washington DC, United States, 2002.

[6] Al-Alawi, B. M., and T. H. Bradley. Review of hybrid, plug-in hybrid, and electric vehicle market modeling Studies. Renewable and Sustainable Energy Reviews, Vol. 21, No. Supplement C, 2013, pp. 190-203.

[7] Litman, T. Autonomous Vehicle Implementation Predictions: Implications for Transport Planning. Presented at Transportation Research Board 94th Annual Meeting, Washington DC, United States, 2015.

[8] Bansal, P., and K. M. Kockelman. Forecasting Americans' long-term adoption of connected and autonomous vehicle technologies. Transportation Research Part A: Policy and Practice, Vol. 95, 2017, pp. 49-63.

[9] Kröger, L., T. Kuhnimhof, and S. Trommer. Modelling the impact of automated driving private autonomous vehicle scenarios for Germany and the US. Presented at European Transport Conference, Barcelona, 2016.

[10] Bhat, C. R., and S. Sen. Household vehicle type holdings and usage: an application of the multiple discrete-continuous extreme value (MDCEV) model. Transportation Research Part B: Methodological, Vol. 40, No. 1, 2006, pp. 35-53.

[11] Auld, J., V. Sokolov, and T. S. Stephens. Analysis of the Effects of Connected-Automated Vehicle Technologies on Travel Demand. Transportation Research Record: Journal of the Transportation Research Board, Vol. 2625, 2017, pp. 1-8.

[12] Childress, S., B. Nichols, B. Charlton, and S. Coe. Using an Activity-Based Model to Explore the Potential Impacts of Automated Vehicles. Transportation Research Record: Journal of the Transportation Research Board, Vol. 2493, 2015, pp. 99-106.

[13] Zhao, Y., K. M. Kockelman. Anticipating the regional impacts of connected and automated vehicle travel in Austin, Texas. Presented at Transportation Research Board 96th Annual Meeting, Washington DC, United States, 2017.

[14] Stephens, T., J. Sullivan, G. A. Keoleian. A Microsimulation of energy demand and greenhouse gas emissions from plug-in hybrid electric vehicle use. Presented at 26th Electric Vehicle Symposium, Los Angeles, California, United States, 2012.

[15] Wang, G., S. Bai, and J. M. Ogden. Identifying contributions of on-road motor vehicles to urban air pollution using travel demand model data. Transportation Research Part D: Transport and Environment, Vol. 14, No. 3, 2009, pp. 168-179.

[16] Mocanu, T., and C. Winkler. Petrol, diesel or electric? An extension of passenger transport models for differentiating car travel. Presented at 6th TRB Innovations in Travel Modeling Conference, Denver, Colorado, 2016. 
[17] Williams, H. C. W. L. On the Formation of Travel Demand Models and Economic Evaluation Measures of User Benefit. Environment and Planning A, Vol. 9, No. 3, 1977, pp. 285-344.

[18] Daly, A., J. Fox, and B. Patruni. Pivoting in travel demand models. Presented at European Transport Conference, Glasgow, 2011.

[19] Winkler, C., and T. Mocanu. Methodology and application of a German National Passenger Transport Model for future transport scenarios. Presented at European Transport Conference, Barcelona, Spain, 2017. 


\section{FIGURES AND TABLES}

TABLE 1 Observed Vehicle Usage Shares in Germany

TABLE 2 Forecasted Overall Car Travel Demand in Germany, 2030

TABLE 3 Forecasted Vehicle Fleet Shares in Germany, 2030

TABLE 4 Overview of the Case Study Results 
TABLE 1 Observed Vehicle Usage Shares in Germany

\begin{tabular}{|c|c|c|c|c|c|c|c|c|c|c|c|c|c|c|}
\hline \multirow[b]{2}{*}{ Distance } & \multirow[b]{2}{*}{ Purpose } & \multicolumn{3}{|c|}{ Small } & \multicolumn{3}{|c|}{ Compact } & \multicolumn{3}{|c|}{ Mid-Size } & \multicolumn{3}{|c|}{ Large } & \\
\hline & & $<4 y r s$ & $4-8 y r s$ & $>8 y r s$ & $<4 y r s$ & 4-8yrs & $>8 y r s$ & $<4 y r s$ & 4-8yrs & $>8 y r s$ & $<4 y r s$ & $4-8 y r s$ & $>8 y r s$ & \\
\hline \multirow{3}{*}{$<20 \mathrm{~km}$} & Commute & 0.072 & 0.078 & 0.133 & 104 & 0.096 & 0.167 & 0.092 & 0.089 & 0.154 & .005 & 0.002 & 0.010 & 1 \\
\hline & Business & 0.059 & 0.051 & 0.089 & 112 & 0.116 & 0.159 & 0.148 & 0.117 & 0.120 & .006 & 0.009 & 0.015 & \\
\hline & Other & 0.067 & 0.069 & 0.097 & .117 & 0.104 & 0.162 & 0.098 & 0.107 & 0.165 & 0.003 & 0.003 & 0.007 & 1 \\
\hline \multirow{3}{*}{$20-50 \mathrm{~km}$} & Commu & 0.085 & 0.081 & 0.104 & 0.115 & 0.118 & 0.155 & 0.097 & 0.099 & 0.131 & 0.004 & 0.004 & 0.006 & 1 \\
\hline & Business & 0.057 & 0.075 & 0.057 & 0.159 & 0.062 & 0.115 & 0.229 & 0.097 & 0.137 & 0.009 & 0.000 & 0.004 & 1 \\
\hline & Other & 0.070 & 0.069 & 0.080 & 0.119 & 0.102 & 0.172 & 0.114 & 0.114 & 0.151 & 0.002 & 0.002 & 0.005 & 1 \\
\hline \multirow{3}{*}{$50-100 \mathrm{~km}$} & Commute & 0.062 & 0.057 & 0.084 & 0.160 & 0.135 & 0.116 & 0.114 & 0.113 & 0.138 & 0.000 & 0.003 & 0.017 & 1 \\
\hline & Business & 0.060 & 0.060 & 0.036 & 0.131 & 0.071 & 0.083 & 0.262 & 0.119 & 0.119 & 0.012 & 0.036 & 0.012 & 1 \\
\hline & Other & 0.076 & 0.069 & 0.057 & 0.116 & 0.079 & 0.169 & 0.113 & 0.143 & 0.146 & 0.004 & 0.008 & 0.019 & 1 \\
\hline \multirow{3}{*}{$100-200 \mathrm{~km}$} & & 0.107 & 0.019 & 0.049 & 0.107 & 0.097 & 0.136 & 0.252 & 0.087 & 0.136 & 0.000 & 0.010 & 0.000 & 1 \\
\hline & Business & 0.050 & 0.033 & 0.017 & 0.150 & 0.083 & 0.050 & 0.283 & 0.083 & 0.183 & 0.050 & 0.017 & 0.000 & 1 \\
\hline & Other & 0.060 & 0.060 & 0.044 & 0.129 & 0.096 & 0.107 & 0.151 & 0.159 & 0.173 & 0.011 & 0.005 & 0.005 & 1 \\
\hline \multirow{3}{*}{$>200 \mathrm{~km}$} & Commute & 0.057 & 0.068 & 0.034 & 0.080 & 0.148 & 0.136 & 0.239 & 0.068 & 0.125 & 0.034 & 0.000 & 0.011 & 1 \\
\hline & Business & 0.000 & 0.000 & 0.022 & 0.200 & 0.000 & 0.044 & 0.467 & 0.089 & 0.156 & 0.000 & 0.022 & 0.000 & 1 \\
\hline & Other & 0.057 & 0.049 & 0.036 & 0.166 & 0.117 & 0.101 & 0.178 & 0.117 & 0.162 & 0.004 & 0.000 & 0.012 & 1 \\
\hline \multicolumn{2}{|c|}{ Vehicle fleet shares } & 0.067 & 0.075 & 0.121 & 0.104 & 0.097 & 0.185 & 0.083 & 0.089 & 0.160 & 0.004 & 0.003 & 0.011 & \\
\hline
\end{tabular}


TABLE 2 Forecasted Overall Car Travel Demand in Germany, 2030

\begin{tabular}{l|c} 
& (commuting trips only) \\
\hline Total number of car trips per average working day & $30.5 \mathrm{~m}$ trips \\
Total car VMT per average working day & $568.1 \mathrm{~m}$ vehicle $\mathrm{km}$ \\
Average trip length & $18.6 \mathrm{~km}$ per trip
\end{tabular}


TABLE 3 Forecasted Vehicle Fleet Shares in Germany, 2030

\begin{tabular}{l|cc|c|r} 
& AV & non-AV & $\boldsymbol{\Sigma}$ & AV available starting \\
\hline Small & 0.000 & 0.227 & 0.227 & n.a. \\
Compact & 0.033 & 0.310 & 0.343 & 2024 \\
Mid-Size & 0.075 & 0.346 & 0.421 & 2021 \\
Large & 0.004 & 0.005 & 0.009 & 2020 \\
\hline $\boldsymbol{\Sigma}$ & 0.112 & 0.888 & 1.000 &
\end{tabular}


TABLE 4 Overview of the Case Study Results

\begin{tabular}{|c|c|c|c|c|c|c|c|c|}
\hline & \multicolumn{2}{|c|}{$\begin{array}{c}\text { Total number of car } \\
\text { trips } \\
{[\mathrm{m}]}\end{array}$} & \multicolumn{2}{|c|}{$\begin{array}{l}\text { Total car VMT } \\
\text { [m veh. km] }\end{array}$} & \multicolumn{2}{|c|}{$\begin{array}{l}\text { Average car trip } \\
\text { length } \\
{[\mathrm{km}]}\end{array}$} & \multicolumn{2}{|c|}{ considers higher AV usage due to: } \\
\hline & AV & non-AV & AV & non-AV & AV & non-AV & AV size and age & vtts reduction \\
\hline A1 & 3.4 & 27.1 & 63.7 & 504.4 & 18.6 & 18.6 & not & not \\
\hline A2 & 4.8 & 25.7 & 102.1 & 466.0 & 21.1 & 18.1 & yes & not \\
\hline A3 & 3.9 & 26.7 & 84.5 & 483.6 & 21.9 & 18.1 & not & yes \\
\hline A4 & 5.5 & 25.1 & 130.5 & 437.6 & 23.9 & 17.5 & yes & yes \\
\hline
\end{tabular}

